\title{
Magnetic resonance imaging brain atrophy assessment in primary age-related tauopathy (PART)
}

\author{
Miguel Quintas-Neves ${ }^{1}$, Merilee A. Teylan², Lilah Besser ${ }^{3}$, João Soares-Fernandes' ${ }^{1}$ Charles N. Mock²,
} Walter A. Kukull ${ }^{2}$, John F. Crary ${ }^{4}$ and Tiago Gil Oliveira ${ }^{1,5,6^{*}}$ (D)

\begin{abstract}
Alzheimer disease (AD) is a neurodegenerative disorder characterized pathologically by the accumulation of amyloid-beta (A $\beta$ ) plaques and tau neurofibrillary tangles (NFTs). Recently, primary age-related tauopathy (PART) has been described as a new anatomopathological disorder where NFTs are the main feature in the absence of neuritic plaques. However, since PART has mainly been studied in post-mortem patient brains, not much is known about the clinical or neuroimaging characteristics of PART. Here, we studied the clinical brain imaging characteristics of PART focusing on neuroanatomical vulnerability by applying a previously validated multiregion visual atrophy scale. We analysed 26 cases with confirmed PART with paired clinical magnetic resonance imaging (MRI) acquisitions. In this selected cohort we found that upon correcting for the effect of age, there is increased atrophy in the medial temporal region with increasing Braak staging $(r=0.3937, p=0.0466)$. Upon controlling for Braak staging effect, predominantly two regions, anterior temporal $(r=0.3638, p=0.0677)$ and medial temporal $(r=0.3836, p=0.053)$, show a trend for increased atrophy with increasing age. Moreover, anterior temporal lobe atrophy was associated with decreased semantic memory/language $(r=-0.5823, p=0.0056$; and $r=-0.6371, p=0.0019$, respectively), as was medial temporal lobe atrophy $(r=-0.4445, p=0.0435)$. Overall, these findings support that PART is associated with medial temporal lobe atrophy and predominantly affects semantic memory/language. These findings highlight that other factors associated with aging and beyond NFTs could be involved in PART pathophysiology.
\end{abstract}

Keywords: Primary age-related tauopathy, Alzheimer disease, MRI, Brain imaging

\section{Introduction}

Degenerative dementias share the common feature of protein misfolding in the brain, resulting in a defining dementia-type abnormal protein with typical topographic deposition. One such example is the accumulation of neurofibrillary tangles (NFTs), composed of hyperphosphorylated tau protein, on a subgroup of neurodegenerative diseases termed tauopathies [14]. Alzheimer disease (AD) is the most studied tauopathy; in this particular case, another hallmark is the presence of extracellular amyloid-beta (A $\beta$ ) plaques and brain atrophy of specific regions, such as the medial temporal

\footnotetext{
* Correspondence: tiago@med.uminho.pt

${ }^{1}$ Division of Neuroradiology, Hospital de Braga, Braga, Portugal

${ }^{5}$ Life and Health Sciences Research Institute (ICVS), School of Medicine,

University of Minho, Campus Gualtar, 4710-057 Braga, Portugal

Full list of author information is available at the end of the article
}

lobe [14]. Following several brain autopsy studies $[28,29]$ from which AD-indistinguishable NFTs were found in the absence of $A \beta$ plaques, the term primary age-related tauopathy (PART) was proposed for this group of patients [10].

The pathological diagnosis of PART requires the presence of tau positive NFT with a Braak stage $\leq$ IV, with minimal deposition beyond medial temporal lobes, and with sparse ("possible PART") to absent ("definite PART") A $\beta$ deposition [10]. Concomitantly, PART can be clinically classified as "asymptomatic" (without cognitive deterioration or dementia) or "symptomatic" (with cognitive deterioration or dementia) [16]. Since PART is essentially a histological designation, there is a need for further studies to advance in vivo diagnostic tools to effectively differentiate it from AD, which could also lead to better selection of patients for clinical trials. Therefore,

(c) The Author(s). 2019 Open Access This article is distributed under the terms of the Creative Commons Attribution 4.0 International License (http://creativecommons.org/licenses/by/4.0/), which permits unrestricted use, distribution, and 
neuropsychological evaluation, cerebrospinal fluid biomarkers assessment and brain magnetic resonance imaging (MRI) are important tools to consider in the characterization of PART.

To clinically differentiate PART from $\mathrm{AD}$, previous studies assessed the relationship between clinical and neuropathologic features. It was observed that Braak staging was associated with an increased odds of having cognitive impairment [7], but comparing to $\mathrm{AD}$, patients with PART were shown to have relative sparing of semantic memory/language [9]. Moreover, it was shown that episodic memory is relatively spared in patients with the moderately severe stratum with a Clinical Dementia Rating $\left(\mathrm{CDR}^{\circ}\right)$ of $0.5-1$, and attention is relatively spared in the most severe stratum with CDR of 2-3 [9]. Consistent findings were described in a clinical-imaging-pathological study that showed that aggregated tau distribution was associated with poorer cognitive performance in "definite" PART patients [20]. Moreover, a recent study showed that autopsy confirmed PART cases with mild cognitive impairment or dementia were clinically diagnosed with $\mathrm{AD}$ greater than $50 \%$ of the time [30]. Therefore, there is a clinical necessity of ante-mortem criteria for the effective diagnosis of PART, for instance, using brain MRI. To date, only one study compared the in vivo brain MRI to neuropathological findings in patients with "definite" PART, which found an association between Braak staging and atrophy of the left head of the hippocampus [20]. Also, it described an antero-posterior gradient of the hippocampus, with relative sparing of the posterior portion, a finding that significantly correlated with the preservation of episodic memories on neuropsychological tests [20]. However, no systematic imaging interpretation method to efficiently use in a clinical setting was proposed.

Therefore, the main goal of our study was to compare in vivo brain MRI findings with neuropathological findings at time of autopsy for people with "definite" PART pathology. Specifically we used previously validated visual atrophy scales that evaluate six different brain regions [13] to assess the potential link of NFT with typical brain imaging pathological patterns, controlling for either Braak staging or age. Moreover, we inquired which neuropsychological domains were specifically correlated with atrophy in the affected brain regions.

\section{Materials and methods \\ Participants}

Cross-sectional data from the National Alzheimer's Coordinating Center's (NACC) Uniform Data Set (UDS) and Neuropathology Data Set (NP) were used. Data contributed to NACC, including demographic, neuropsychological, clinical, and diagnostic data on participants with normal cognition, mild cognitive impairment, and dementia, were collected since 2005 from approximately 30 past and present Alzheimer's Disease Centers (ADC) in the United States. The UDS and NP data have been meticulously described $[4,5,8,23]$. We used data collected between September 2005 and March 2018. All participants provided written informed consent at each ADC.

\section{Selection criteria}

From the total number of UDS participants as of the March 2018 data freeze $(n=37,568)$, only those with neuropathological data $(n=5135)$, most specifically neuritic plaque (NP) data $(n=5110)$ were enrolled. We excluded participants: (a) with neuropathological evidence of frontotemporal lobar degeneration, Lewy bodies, amyotrophic lateral sclerosis, prion disease, or argyrophilic grains; (b) with clinical evidence of dementia with Lewy bodies, Parkinson disease, Down syndrome, Huntington disease, prion disease, corticobasal degeneration, or progressive supranuclear palsy. With the application of these exclusion criteria, 2350 participants remained. Considering only those with "definite" PART (defined as no NP $n=394$ ) and availability of MRI scans, 33 participants were considered. Five patients with other lesions that biased volumetric interpretation (e.g., brain tumors) were excluded. Two patients less than 40 years old were excluded, in order to obtain a more homogeneous cohort in terms of age. Therefore, our cohort was composed of 26 patients.

\section{Neuropathology data}

Neuropathological data was collected by the ADCs by using a standardized Neuropathology Form on UDS patients who died and consented to autopsy and neuropathologic examination. Details on brain tissue preparation and staining within the NACC NP dataset have been previously described [8]. This data was used to establish the participants with "definite" PART, and to obtain the Braak stage for neurofibrillary degeneration.

\section{Brain MRI data}

MR imaging examinations were performed mostly on $1.5 \mathrm{~T}$ or $3 \mathrm{~T}$ scanners, both from Philips ${ }^{\circ}$, Siemens ${ }^{\circ}$ or $\mathrm{GE}^{\bullet}$ manufacturers. Several imaging protocols were used by the different centres; we always used T1-weighted sequences in order to establish the degree of brain atrophy - spin-echo (SE) or magnetization-prepared rapid gradient-echo (MPRAGE) sequences.

\section{Image analysis}

We used a group of validated MRI visual rating scales [13] in order to assess brain atrophy on the following regions: anterior cingulate, orbito-frontal, anterior temporal, fronto-insular, medial temporal, and posterior regions. Briefly, as previously described by the simplified 
version of these scales: orbito-frontal and anterior cingulate regions were rated on the first anterior slice where the corpus callosum becomes visible, both from " $0-3$ ", where " 0 " is representing no atrophy; the fronto-insular was rated over three slices, starting on the first anterior slice where the anterior cingulate becomes visible and moving posteriorly, from " $0-3$ "; the anterior temporal was rated at the level of the temporal pole, just anterior to where the "temporal stem" connects the frontal and temporal lobes, from " $0-4$ "; the medial temporal was rated according to the medial temporal lobe atrophy (MTA) score - performed on the hippocampus at the level of the anterior pons, from " $0-4$ "; the posterior region was rated according to the four-point posterior atrophy scale described by Koedam, from "0-3" [21] overall score based on the presence of atrophy in sagittal (widening of the posterior cingulate and parietooccipital sulcus, and atrophy of the precuneus on left and right by considering paramedian sagittal images), axial (widening of the posterior cingulate sulcus and sulcal dilatation in parietal lobes on axial images) and coronal (widening of the posterior cingulate sulcus and parietal lobes on coronal images) orientations, assessed for left and right separately [13]. Two independent classifiers with neuroradiology experience were responsible for rating the images. For each brain region scale, an average of both hemispheres was considered and an average of both classifiers was used. In this specific cohort no significant asymmetric atrophy was observed (Additional file 1: Figure S1). In order to aid the rating process, reference images for each rating scale were provided to the classifiers based on Harper et al. [13]. Atrophy classification data is summarized in Additional file 1 : Table S1. For Fig. 4, scale values were then converted to percentage values, from $0 \%$, as no atrophy, to $100 \%$, as maximal atrophy value considered for each scale. Images were visualized in Horos $^{\bullet}$ imaging software version 3.0.1.

\section{Neuropsychological data}

Several tests of memory, executive function, language and processing speed were considered in our analysis. Executive function was assessed by the Trail Making Test (TMT) A and B, which globally tests attention, visual scanning and search skills, and psychomotor speed and coordination [26]; TMT A can independently assess processing speed, while TMT B assesses set switching; on both parts of this test (i.e., A and B), the total number of seconds to complete the test, the number of commission errors, and number of correct lines were recorded; the Wechsler Adult Intelligence Scale digit symbol test was also considered to provide an estimate of processing speed [31]. Semantic memory/language was assessed by category (vegetables and animals) verbal fluency [22], consisting of a test on registering the total number of vegetables and animals named in $60 \mathrm{~s}$; the Boston naming test [11], which also assesses the effect of language function, more precisely the confrontational word retrieval, was included in this evaluation, and consisted of showing pictures (up to 60) to the patient, and wait up to $20 \mathrm{~s}$ for the patients to name them. Attention and working memory was evaluated by Digit span forwards and backwards test [18], consisting on registering the ability of recalling a sequence of numbers shown, and the total length of numbers successfully achieved. Episodic memory was assessed by Current Logical Memory 1A Story Units Recalled [1], for the total number of items recalled. Mini-Mental State Examination [12] was performed as a brief cognitive screening instrument that provides a measure of global cognition. Concerning the data used for this study, the average delay between MRI and neuropsychological data acquisition was 2.7 years. All valid and available neuropsychological data retrieved from NACC concerning the PART population under study was included for analysis and is summarized in Additional files 1: Table S2.

\section{Statistical analysis}

In order to assess the rating acuity, Spearman's correlation coefficient was calculated for the pair of classifiers per each region and it was confirmed that there was a statistically significant association between both (Additional file 1: Table S3). Residuals were calculated for brain regional atrophy scores controlling for either age or Braak staging. These were then plotted independently to Braak staging and age, respectively. Regional brain atrophy scores were also plotted for each of neuropsychological test considered and only significant associations were represented. Pearson correlation coefficients were calculated $(r)$ and statistically significant values were considered for $p<0.05$. SPSS $^{\circ}$ Statistics version 25, and $\mathrm{GraphPad}^{\circ}$ Software version 8.0.0 were used for all analyses.

\section{Results}

Twenty-six participants were considered for this study. Table 1 summarizes the demographic characteristics of the enrolled participants. Of the 26 "definite" PART patients, $17(65.4 \%)$ were male. The mean age at the time of MRI examination was 80.6 years (range: $51-99$ ), and the mean age of death was 83.5 years (range: 51-101). The mean Braak stage was 1.5 (range: 0-4).

In this specific cohort, even though there was no significant correlation between age and Braak stage $(r=-$ $0.03079, p=0.8813$ ) (Fig. 1a) the majority of cases had some degree of regional atrophy (Fig. 1b), and no significant changes were observed between men and women (Additional files 1: Figure S2). Upon correction for age, the relative percentage of atrophy of the medial temporal lobe significantly correlated with Braak staging $(r=$ 
Table 1 Demographic characteristics of definite PART patients

\begin{tabular}{ll}
\hline & PART population $(N=26)$ \\
\hline Age MRI & $80.6(51-99)$ \\
Age of death & $83.5(51-101)$ \\
Difference age MRI to death & $2.96(1-7)$ \\
Braak stage & $1.5(0-4)$ \\
Sex & \\
$\quad$ Male & $17(65.4 \%)$ \\
Female & $9(34.6 \%)$ \\
\hline
\end{tabular}

The table shows the summarized demographic characteristics of a cohort of 26 anatomopathological confirmed PART cases. Age MRI is the subject age when brain imaging acquisition was performed and is reported in years as a continuous variable with average, minimum and maximum values represented. Age of death is reported in years as a continuous variable with mean, minimum and maximum values represented. Braak stage is represented as categorical values based on an anatomopathological validated staging scale with mean, minimum and maximum values represented. Sex is differentially represented as male and female with frequency and relative percent values for each

0.3937; $p=0.0466$ ) (Fig. 2e). No statistically significant results of regional brain atrophy were found for the other regions evaluated (Fig. 2). Upon correction for Braak staging, we observed a trend to increasing atrophy with age, predominantly on the anterior temporal $(r=$ 0.3638, $p=0.0677$ ) (Fig. 3c) and medial temporal $(r=$ $0.3836, p=0.053$ ) regions (Fig. 3e). Moreover, when we compared cases Braak $\leq 1$ with Braak $\geq 2$ we observed significant increased atrophy score in the medial temporal region (Additional file 1: Figure S3).

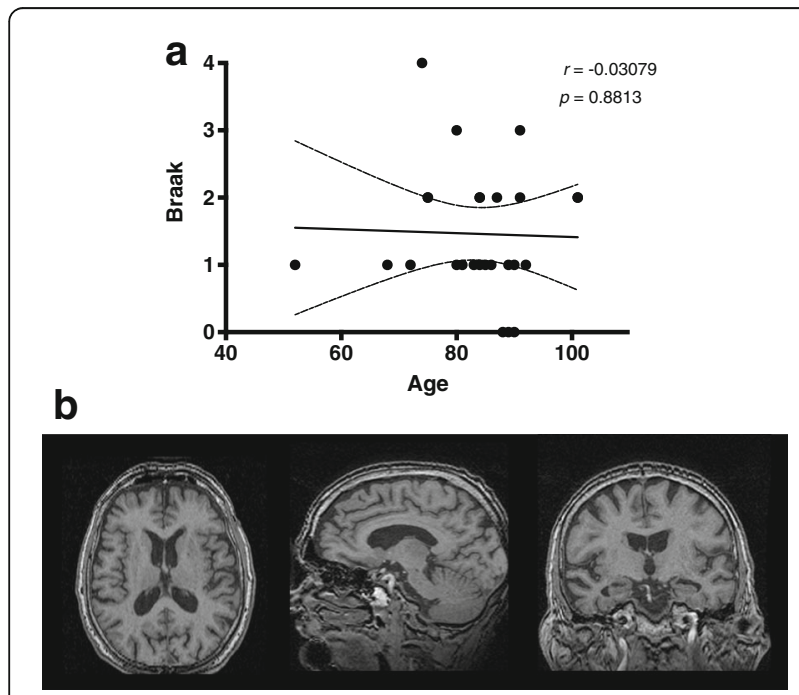

Fig. 1 Distribution of cases from a definite PART cohort based on age and Braak staging. a Age in years is represented based on age of death of the subjects. Braak staging is represented as categorical values based on an anatomopathological validated staging scale. b Representative case of a 78 year-old male from the PART cohort. Axial, sagittal and coronal MRI T1-weighed images are shown
From the 26 cases, the majority of them also performed an extensive neuropsychological battery testing. Considering this sample, we checked for an association between regional brain atrophy and performance in each of the cognitive domains assessed. We observed that atrophy of the anterior temporal lobe was associated with decreased semantic memory/language, given by the animals and vegetables naming tests $(r=-0.5823$, $p=0.0056$; and $r=-0.6371, p=0.0019$, respectively) (Fig. 4a, b), as was atrophy of the medial temporal lobe, given by the vegetables naming test $(r=-$ 0.4445, $p=0.0435$ ) (Fig. 4c).

\section{Discussion}

The main goal of this study was to compare the in vivo brain MRI findings to neuropathological findings at autopsy for patients identified with "definite" PART pathology. More specifically, we assessed the effects of Braak staging and age on PART brain imaging associated atrophy patterns and its potential implications for specific neuropsychological deficits. Our results showed an association between Braak staging and atrophy of the medial temporal lobe on "definite" PART patients (i.e., brains without neuritic plaques) upon correction for age. This main finding was consistent with one described by another imaging study, on which left hippocampal volume was found to be decreased in PART [20]. It is also consistent with previous anatomopathological studies describing PART, where NFTs accumulation is predominantly confined to the temporal lobe [10]. Importantly, medial temporal lobe atrophy is commonly found in typical AD, namely with decreased hippocampal volume [25]. While at the imaging level this observation does not help completely to distinguish PART from early stage $\mathrm{AD}$, it might distinguish it from more advanced cases of $\mathrm{AD}$ where atrophy is extensive in other brain regions. Given the sparsity of PART patients with Braak stages III and IV, this is an assumption future studies with more patients on these advanced stages should address.

Our findings partly explain why many PART cases identified post-mortem were clinically diagnosed as AD [30]. The combination of other features, such as neuropsychological specific presentation, CSF signatures or PET could lead to a more fine-tuned distinction between PART and AD.

Various other observations support PART as a separate pathological entity: the absence of an association between PART and APOE \&4 allele, the strongest risk factor for AD [3, 15]; "ghost" tangles, i.e. extracellular tangles, are more frequently found in PART than AD patients [17]; and patients diagnosed with PART are frequently in the 8th-9th decades, as was found in our study, making it highly improbable that $\mathrm{A} \beta$ deposition would only start 

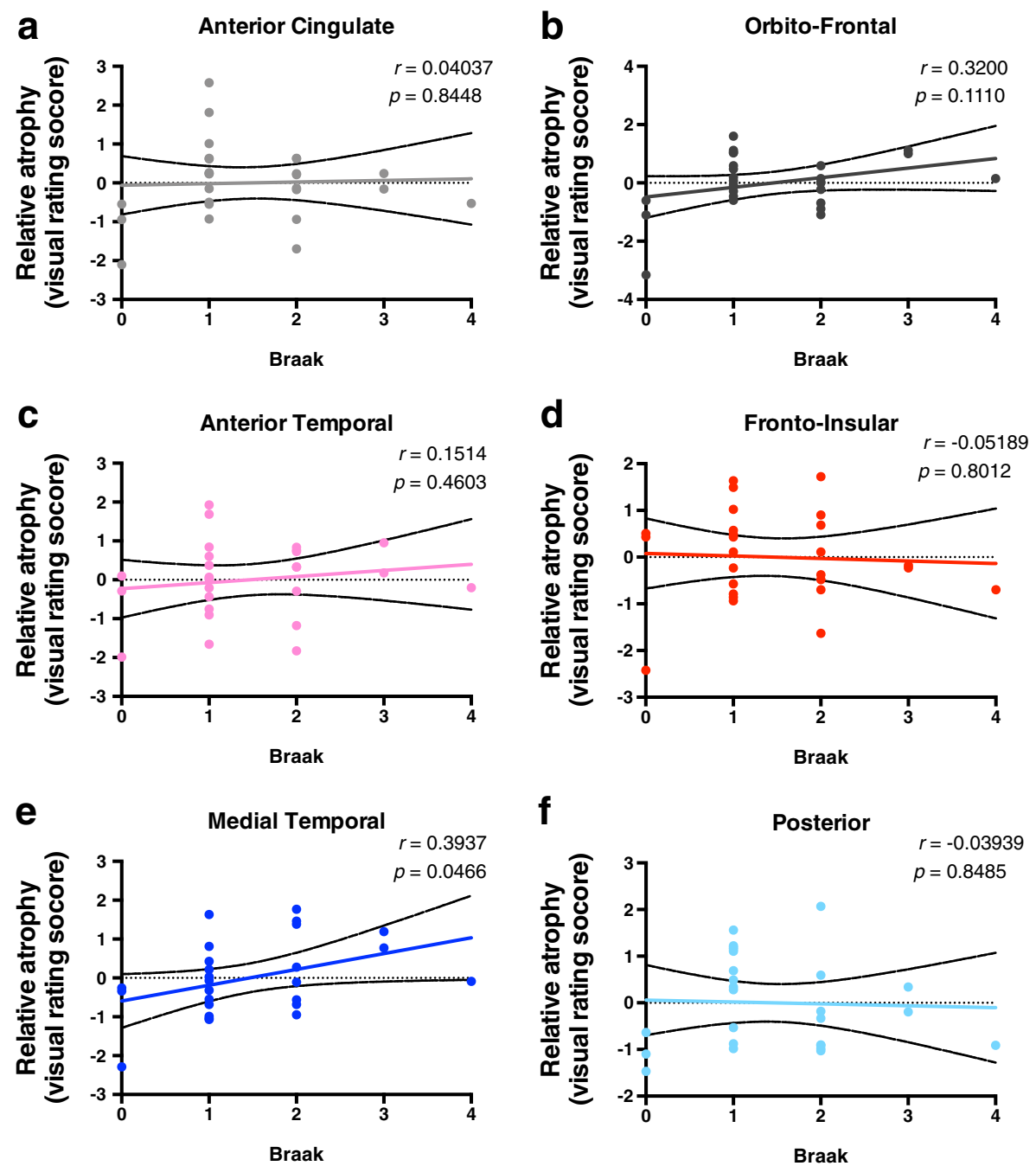

Fig. 2 Medial temporal lobe atrophy correlates with increasing Braak staging. The values represented are residuals corrected for age, with Pearson correlation analysis between Braak staging and atrophy of different brain regions based on a previously validated imaging rating scale. The regions evaluated are $\mathbf{a}$ anterior cingulate, $\mathbf{b}$ orbito-frontal, $\mathbf{c}$ anterior temporal, $\mathbf{d}$ fronto-insular, $\mathbf{e}$ medial temporal and $\mathbf{f}$ posterior brain regions. Statistical significance was considered as $p<0.05$

then [20]. In fact, our results support the hypothesis of PART being a form of pathological brain aging, with preferential atrophy in the medial temporal lobes.

After controlling for Braak staging, our observations indicate that, in this selected cohort, aging leads to a higher degree of atrophy with predominance over the anterior and medial temporal lobe regions - a fact that is consistent with previous reported effects of aging [27]. One hypothesis is that other neuropathological factors associated with aging and beyond NFTs could be contributing to this regional temporal atrophy, one possibility being transactive response DNA-binding protein 43 (TDP-43). Interestingly, a recently described neuropathologic entity, limbic-predominant agerelated TDP-43 encephalopathy (LATE), which is characterized by TDP- 43 accumulation in the limbic system, can also present with dementia symptoms and brain atrophy, with potential involvement of the temporal lobe [24]. Remarkably, not only was TDP-43 pathology associated with greater atrophy in the medial temporal lobe regions in PART cases [19], but also independently of other neuropathological conditions [6]. Therefore, future studies should address the independent or cumulative impact of $\mathrm{A} \beta$, tau and TDP-43 on brain regional atrophy, using $\mathrm{AD}$, PART and LATE cohorts. Even though major vascular lesions were excluded after clinical neuroradiologist assessment, it is still possible that finer microvascular lesions, which are partially identified as white matter T2 hyperintensities, could be another potential contributing factor addressed in future studies. 
a
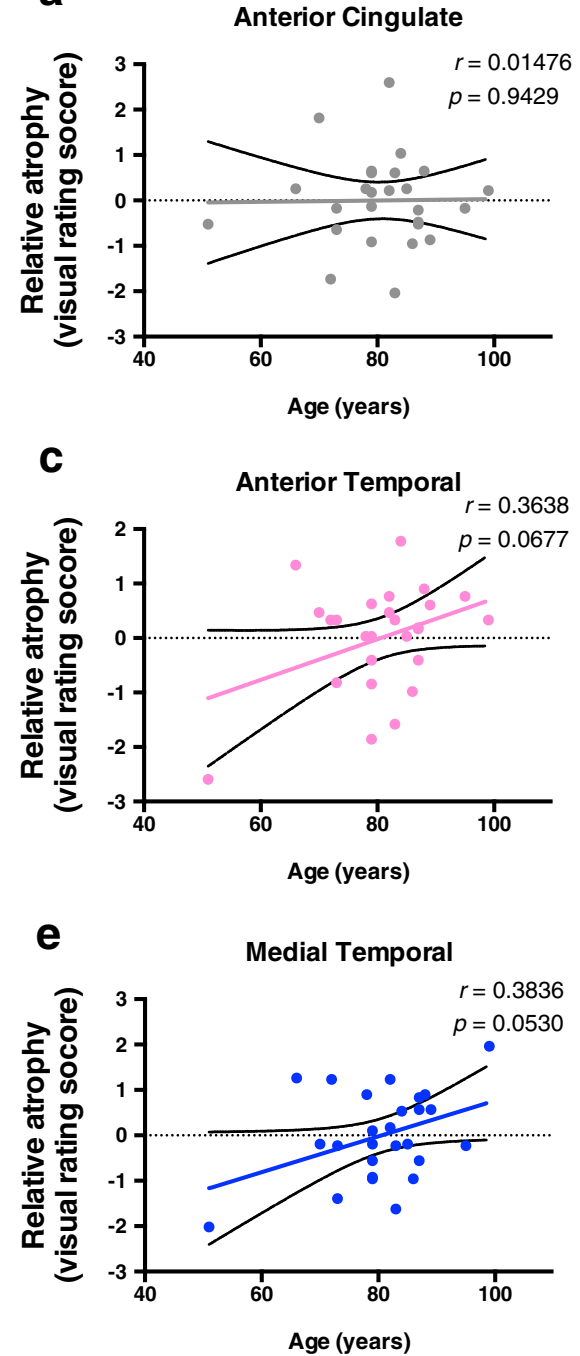

b

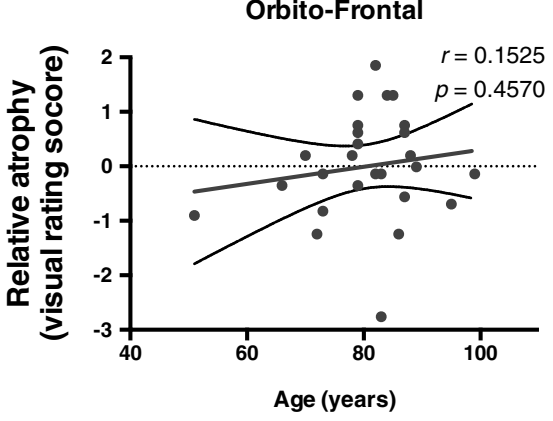

d

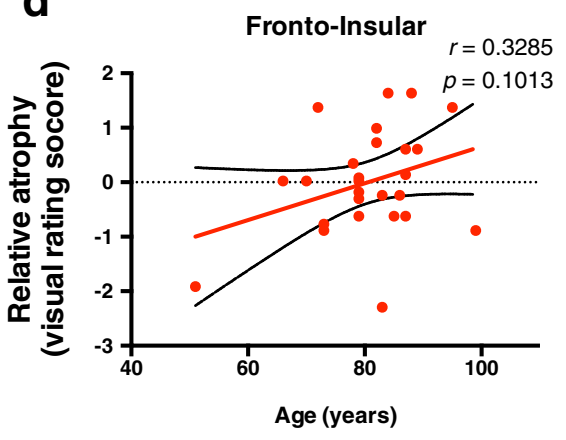

f

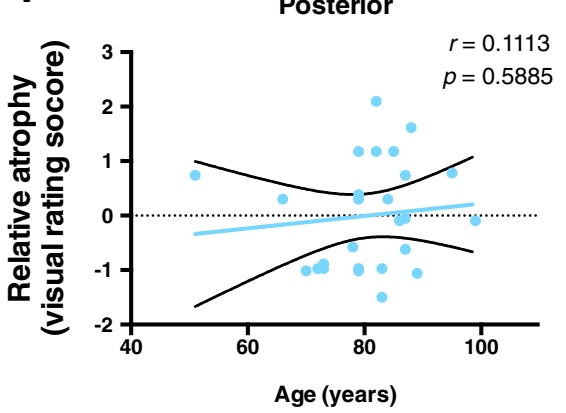

Fig. 3 Anterior and Medial temporal lobe atrophy increases with increasing age. The values represented are residuals corrected for Braak staging, with Pearson correlation analysis between age and atrophy of different brain regions based on a previously validated imaging rating scale. The regions evaluated are $\mathbf{a}$ anterior cingulate, $\mathbf{b}$ orbito-frontal, $\mathbf{c}$ anterior temporal, $\mathbf{d}$ fronto-insular, e medial temporal and $\mathbf{f}$ posterior brain regions. Since statistical significance was considered as $p<0.05$, no significant correlations were found, although both anterior temporal $(r=0.3638, p=$ 0.0677), and medial temporal $(r=0.3836, p=0.053)$ regions were found to be close to this statistical threshold

We further inquired as to which neuropsychological domains could be impaired with the observed atrophy in the temporal lobe. Indeed, anterior temporal lobe atrophy significantly correlated with worse performance on animals and vegetables naming, i.e., semantic memory/language domain tests, as also did medial temporal lobe atrophy with vegetables naming. These findings are consistent with a previous study that stated the importance of temporal lobe on semantic verbal fluency [2]. Moreover, our results are consistent with a previous study that showed predominant anterior hippocampus atrophy on patients with PART, which also presented semantic memory/language domain deficits [20]. Another key point is that, despite using a different brain atrophy assessment methodology (i.e. visual rating scales), our observations are somewhat consistent with previous reports using voxel-based morphometry [20]. Also, we believe our study provides evidence for the use of a clinical settingapplicable visual rating scale that can efficiently assess brain regional atrophy by a trained neuroradiologist. This could be paramount to routine clinical practice, given the limited time and resources to continuously post-process volumetric acquisitions. Since another study found that semantic memory/language was relatively preserved in PART compared to AD patients [9], future imaging studies should tackle the question of potential different patterns of atrophy in PART and AD, and its correlation with neuropsychological presentation. 
While this study had some important strengths, such as the confirmed neuropathologic data and the usage of an easy to apply and interpret atrophy rating score, there are some important limitations. First, it was based on a small, convenience, autopsy-based sample, a factor that limits its generalizability to other

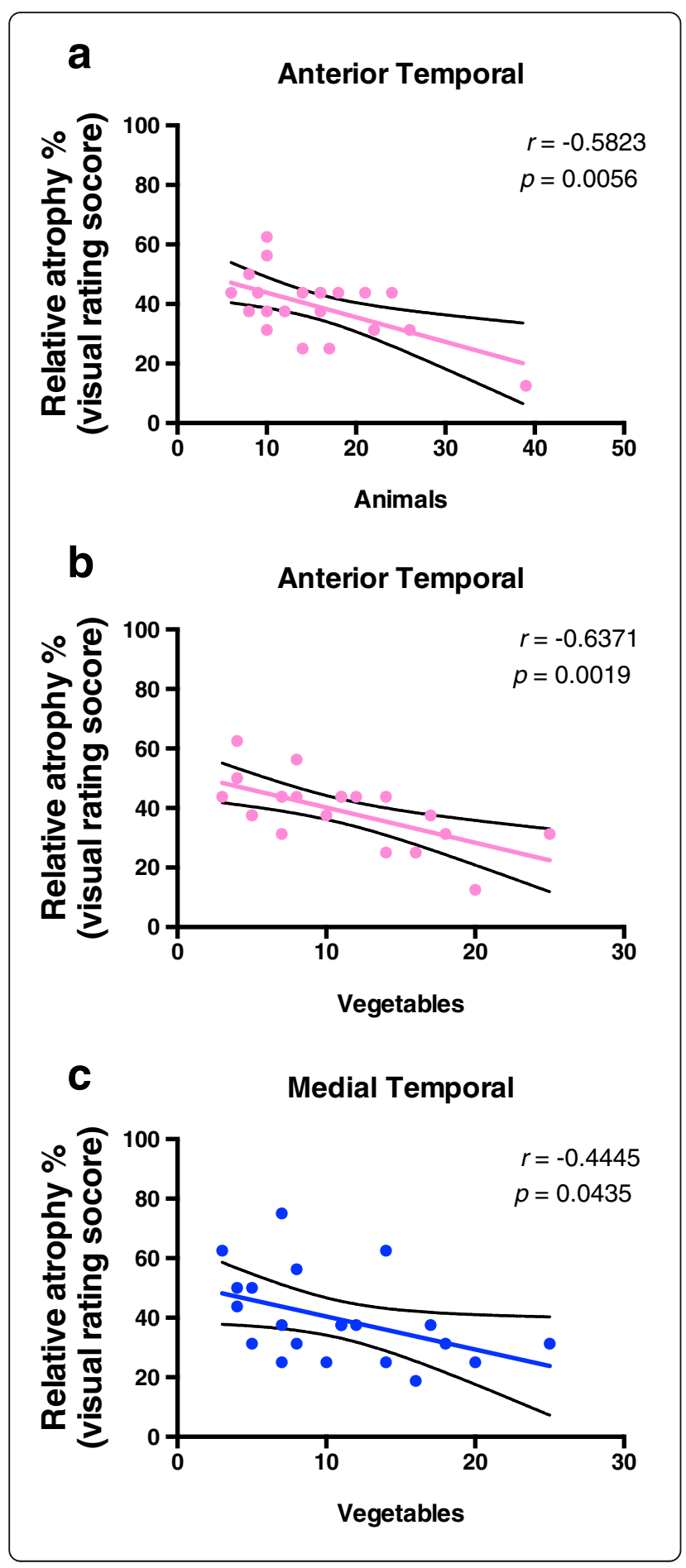

Fig. 4 Semantic memory/language impairment correlates with anterior and medial temporal atrophy. Pearson correlation analysis of neuropsychological test performance versus temporal brain atrophy regions evaluated with a previously validated imaging rating scale for patients with definite PART was performed and only the statistically significant correlations are shown $(p<0.05)$. "Vegetables" represents the number of vegetables a subject can name in 1 min. "Animals" represents the number of animals a subject can name in $1 \mathrm{~min}$. a Anterior temporal lobe atrophy is associated with decreased semantic memory/language, given by the animals $(r=-0.5823, p=0.0056)$ and $\mathbf{b}$ vegetables naming tests $(r=-0.6371, p=0.0019)$. $\mathbf{c}$ Medial temporal lobe atrophy is also associated with decreased semantic memory/language, given by the vegetables naming test $(r=-0.4445, p=0.0435)$

populations. The NACC database, in general, has limitations of its generalizability, as the participants tend to be more white and more affluent than the population as a whole. However, there was no additional selection bias in choosing the participants for this study, as we chose all eligible participants (i.e. people who had neuropathologically defined PART and who had an MRI available). Second, despite using validated MRI rating scales on patients with dementia, qualitative assessments and rating are almost invariably associated with inter-observer variability. However, given that observers applying these ratings should have several years of neuroradiology experience, we believe that in the clinical setting this could be overcome. Third, despite always using T1-weighted images on MRI visual rating, not all were volumetric acquisitions, introducing a source of variability in this study. Fourth, the variability in scanner manufacturers and field strengths used on the acquisition of images is also a potential source of variability in the interpretation and rating of the brain regional atrophy. Finally, another limitation is that the subset of subjects that were included in this study had incomplete information on other co-morbidities that could be associated with brain atrophy, such as hypertension or diabetes mellitus, or on other neuropathologic features, such as TDP-43 pathology.

\section{Conclusion}

In summary, our findings provide evidence that in "definite" PART patients, i.e. brains with tau deposition without neuritic plaques, there is a correlation between atrophy of the medial temporal lobe and Braak staging. Moreover, since we show that aging leads to temporal lobe atrophy, independently of Braak, other factors beyond tau tangles could be contributing to atrophy. Finally, we provide evidence of a potential deficit on semantic memory/language domain in these patients. Future studies with volumetric imaging acquisitions should focus on differences in brain regional atrophy between PART and AD patients. 


\section{Supplementary information}

Supplementary information accompanies this paper at https://doi.org/10. 1186/s40478-019-0842-z.

Additional file 1. Table S1. Atrophy Classification in PART population. Table S2. Neuropsychological evaluation in PART population. Table S3. Inter-rater analysis. Figure S1. No asymmetric atrophy observed. Comparison between right and left hemisphere regional atrophy ratings was performed. The regions evaluated are $\mathbf{a}$ anterior cingulate, $\mathbf{b}$ orbitofrontal, c anterior temporal, $\mathbf{d}$ fronto-insular, e medial temporal and $\mathbf{f}$ posterior brain regions. Figure S2. No sex differences in atrophy. Comparison of regional atrophy ratings was performed between men and women cases. The regions evaluated are $\mathbf{a}$ anterior cingulate, $\mathbf{b}$ orbito-frontal, $\mathbf{c}$ anterior temporal, $\mathbf{d}$ fronto-insular, e medial temporal and $\mathbf{f}$ posterior brain regions. Figure S3. Higher Braak score shows higher atrophy in the Medial Temporal region. Comparison between cases with Braak $\leq 1$ and Braak $\geq 2$. The regions evaluated are $\mathbf{a}$ anterior cingulate, $\mathbf{b}$ orbito-frontal, c anterior temporal, $\mathbf{d}$ fronto-insular, e medial temporal and $\mathbf{f}$ posterior brain regions. ${ }^{*} p<0.05$.

\section{Authors' contributions}

TGO and JFC conceived the idea. TGO, JFC, CM, LB, MAT and MQ designed and planned the analysis. TGO, JS and MQ performed neuroimaging analysis. WAK, CM, LB and MAT contributed with subject data. TGO and MQ conducted data analysis and wrote the manuscript. All authors reviewed and corrected the manuscript. All authors read and approved the final manuscript.

\section{Acknowledgements}

The NACC database is funded by NIA/NIH Grant U01 AG016976. NACC data are contributed by the NIA-funded ADCs: P30 AG019610 (PI Eric Reiman, MD), P30 AG013846 (PI Neil Kowall, MD), P30 AG062428-01 (PI James Leverenz, MD) P50 AG008702 (PI Scott Small, MD), P50 AG025688 (PI Allan Levey, MD, PhD), P50 AG047266 (PI Todd Golde, MD, PhD), P30 AG010133 (PI Andrew Saykin, PsyD), P50 AG005146 (PI Marilyn Albert, PhD), P30 AG062421-01 (PI Bradley Hyman, MD, PhD), P30 AG062422-01 (PI Ronald Petersen, MD, PhD), P50 AG005138 (PI Mary Sano, PhD), P30 AG008051 (PI Thomas Wisniewski, MD), P30 AG013854 (PI Robert Vassar, PhD), P30 AG008017 (PI Jeffrey Kaye, MD), P30 AG010161 (PI David Bennett, MD), P50 AG047366 (PI Victor Henderson, MD, MS), P30 AG010129 (PI Charles DeCarli, MD), P50 AG016573 (PI Frank LaFerla, PhD), P30 AG062429-01(PI James Brewer, MD, PhD), P50 AG023501 (PI Bruce Miller, MD), P30 AG035982 (PI Russell Swerdlow, MD), P30 AG028383 (PI Linda Van Eldik, PhD), P30 AG053760 (PI Henry Paulson, MD, PhD), P30 AG010124 (PI John Trojanowski, MD, PhD), P50 AG005133 (PI Oscar Lopez, MD), P50 AG005142 (PI Helena Chui, MD), P30 AG012300 (PI Roger Rosenberg, MD), P30 AG049638 (PI Suzanne Craft, PhD), P50 AG005136 (PI Thomas Grabowski, MD), P30 AG062715-01 (PI Sanjay Asthana, MD, FRCP), P50 AG005681 (PI John Morris, MD), P50 AG047270 (PI Stephen Strittmatter, MD, PhD). NIH grants to JFC (R01AG054008, R01NS095252, R01AG062348, RF1AG060961), the Tau Consortium, and Alzheimer's Association (NIRG- 469 15-363188).

\section{Availability of data and materials}

The datasets used and/or analyzed during the current study are available upon request on the NACC database.

\section{Ethics approval and consent to participate}

Cross-sectional data from the NACC UDS and NP data set were used. All participants provided written informed consent at each ADC.

\section{Consent for publication}

Not applicable.

\section{Competing interests}

The authors declare that they have no competing interests.

\section{Author details}

${ }^{1}$ Division of Neuroradiology, Hospital de Braga, Braga, Portugal. ${ }^{2}$ Department of Epidemiology, National Alzheimer's Coordinating Center, University of Washington, Seattle, Washington, USA. ${ }^{3}$ School of Urban and Regional
Planning, Institute for Human Health and Disease Intervention, Florida Atlantic University, Boca Raton, Florida, USA. ${ }^{4}$ Department of Pathology, Nash Family Department of Neuroscience, Friedman Brain Institute, Ronald M. Loeb Center for Alzheimer's Disease, Icahn School of Medicine at Mount Sinai, New York, NY, USA. ${ }^{5}$ Life and Health Sciences Research Institute (ICVS), School of Medicine, University of Minho, Campus Gualtar, 4710-057 Braga, Portugal. ${ }^{6}$ ICVS/3B's_-PT Government Associate Laboratory, Braga/Guimarães, Portugal.

Received: 24 September 2019 Accepted: 7 November 2019

Published online: 09 December 2019

\section{References}

1. Abikoff H, Alvir J, Hong G, Sukoff R, Orazio J, Solomon S, Saravay S (1987) Logical memory subtest of the Wechsler memory scale: age and education norms and alternate-form reliability of two scoring systems. J Clin Exp Neuropsychol 9:435-448. https://doi.org/10.1080/01688638708405063

2. Baldo JV, Schwartz S, Wilkins D, Dronkers NF (2006) Role of frontal versus temporal cortex in verbal fluency as revealed by voxel-based lesion symptom mapping. J Int Neuropsychol Soc 12:896-900. https://doi.org/10. 1017/\$1355617706061078

3. Bancher C, Egensperger R, Kosel S, Jellinger K, Graeber MB (1997) Low prevalence of apolipoprotein $E$ epsilon 4 allele in the neurofibrillary tangle predominant form of senile dementia. Acta Neuropathol 94:403-409

4. Beekly DL, Ramos EM, Lee WW, Deitrich WD, Jacka ME, Wu J, Hubbard JL, Koepsell TD, Morris JC, Kukull WA et al (2007) The National Alzheimer's coordinating center (NACC) database: the uniform data set. Alzheimer Dis Assoc Disord 21:249-258. https://doi.org/10.1097/WAD.0b013e318142774e

5. Beekly DL, Ramos EM, van Belle G, Deitrich W, Clark AD, Jacka ME, Kukull WA, Centers NI-AsD (2004) The National Alzheimer's coordinating center (NACC) database: an Alzheimer disease database. Alzheimer Dis Assoc Disord 18:270-277

6. Bejanin A, Murray ME, Martin P, Botha H, Tosakulwong N, Schwarz CG, Senjem ML, Chetelat G, Kantarci K, Jack CR et al (2019) Antemortem volume loss mirrors TDP-43 staging in older adults with non-frontotemporal lobar degeneration. Brain 142:3621-3635. https://doi.org/10.1093/brain/awz277

7. Besser LM, Crary JF, Mock C, Kukull WA (2017) Comparison of symptomatic and asymptomatic persons with primary age-related tauopathy. Neurology 89:1707-1715. https://doi.org/10.1212/WNL.0000000000004521

8. Besser LM, Kukull WA, Teylan MA, Bigio EH, Cairns NJ, Kofler JK, Montine TJ, Schneider JA, Nelson PT (2018) The revised National Alzheimer's coordinating Center's neuropathology form-available data and new analyses. J Neuropathol Exp Neurol 77:717-726. https://doi.org/10.1093/ jnen/nly049

9. Besser LM, Mock C, Teylan MA, Hassenstab J, Kukull WA, Crary JF (2019) Differences in cognitive impairment in primary age-related Tauopathy versus Alzheimer disease. J Neuropathol Exp Neurol 78:219-228. https://doi.org/10.1093/jnen/nly132

10. Crary JF, Trojanowski JQ, Schneider JA, Abisambra JF, Abner EL, Alafuzoff I, Arnold SE, Attems J, Beach TG, Bigio EH et al (2014) Primary age-related tauopathy (PART): a common pathology associated with human aging. Acta Neuropathol 128:755-766. https://doi.org/10.1007/s00401-014-1349-0

11. Edith Kaplan HG, Weintraub S (1983) The Boston naming test. Lea \& Febiger, City, Philadelphia

12. Folstein MF, Folstein SE, McHugh PR (1975) "Mini-mental state". A practical method for grading the cognitive state of patients for the clinician. J Psychiatr Res 12:189-198

13. Harper L, Fumagalli GG, Barkhof F, Scheltens P, O'Brien JT, Bouwman F, Burton EJ, Rohrer JD, Fox NC, Ridgway GR et al (2016) MRI visual rating scales in the diagnosis of dementia: evaluation in 184 post-mortem confirmed cases. Brain 139:1211-1225. https://doi.org/10.1093/brain/aww005

14. Hyman BT, Phelps CH, Beach TG, Bigio EH, Cairns NJ, Carrillo MC, Dickson DW, Duyckaerts C, Frosch MP, Masliah E et al (2012) National Institute on Aging-Alzheimer's Association guidelines for the neuropathologic assessment of Alzheimer's disease. Alzheimers Dement 8:1-13. https://doi.org/10.1016/j.jalz.2011.10.007

15. Ikeda K, Akiyama H, Arai T, Sahara N, Mori H, Usami M, Sakata M, Mizutani T, Wakabayashi K, Takahashi H (1997) A subset of senile dementia with high incidence of the apolipoprotein E epsilon2 allele. Ann Neurol 41:693-695. https://doi.org/10.1002/ana.410410522 
16. Jellinger KA, Alafuzoff I, Attems J, Beach TG, Cairns NJ, Crary JF, Dickson DW, Hof PR, Hyman BT, Jack CR Jr et al (2015) PART, a distinct tauopathy, different from classical sporadic Alzheimer disease. Acta Neuropathol 129: 757-762. https://doi.org/10.1007/s00401-015-1407-2

17. Jellinger KA, Attems J (2007) Neurofibrillary tangle-predominant dementia: comparison with classical Alzheimer disease. Acta Neuropathol 113:107-117. https://doi.org/10.1007/s00401-006-0156-7

18. Jensen AR, Figueroa RA (1975) Forward and backward digit span interaction with race and IQ: predictions from Jensen's theory. J Educ Psychol 67:882-893

19. Josephs KA, Murray ME, Tosakulwong N, Weigand SD, Knopman DS, Petersen RC, Jack CR Jr, Whitwell JL, Dickson DW (2019) Brain atrophy in primary age-related tauopathy is linked to transactive response DNAbinding protein of $43 \mathrm{kDa}$. Alzheimers Dement 15:799-806. https://doi.org/ 10.1016/j.jalz.2019.03.003

20. Josephs KA, Murray ME, Tosakulwong N, Whitwell JL, Knopman DS, Machulda MM, Weigand SD, Boeve BF, Kantarci K, Petrucelli L et al (2017) Tau aggregation influences cognition and hippocampal atrophy in the absence of beta-amyloid: a clinico-imaging-pathological study of primary age-related tauopathy (PART). Acta Neuropathol 133:705-715. https://doi.org/10.1007/s00401-017-1681-2

21. Koedam EL, Lehmann M, van der Flier WM, Scheltens P, Pijnenburg YA, Fox N, Barkhof F, Wattjes MP (2011) Visual assessment of posterior atrophy development of a MRI rating scale. Eur Radiol 21:2618-2625. https://doi.org/ 10.1007/s00330-011-2205-4

22. Lezak M, Howieson D, Loring D, Hannay H, Fischer J. (2004) Neuropsychological assessment. New York: Oxford University Press Inc

23. Morris JC, Weintraub S, Chui HC, Cummings J, Decarli C, Ferris S, Foster NL, Galasko D, Graff-Radford N, Peskind ER et al (2006) The uniform data set (UDS): clinical and cognitive variables and descriptive data from Alzheimer disease centers. Alzheimer Dis Assoc Disord 20:210-216. https://doi.org/10 1097/01.wad.0000213865.09806.92

24. Nelson PT, Dickson DW, Trojanowski JQ, Jack CR, Boyle PA, Arfanakis K, Rademakers R, Alafuzoff I, Attems J, Brayne C et al (2019) Limbic-predominant age-related TDP-43 encephalopathy (LATE): consensus working group report Brain 142:1503-1527. https://doi.org/10.1093/brain/awz099

25. Pereira JB, Cavallin L, Spulber G, Aguilar C, Mecocci P, Vellas B, Tsolaki M, Kloszewska I, Soininen H, Spenger C et al (2014) Influence of age, disease onset and ApoE4 on visual medial temporal lobe atrophy cut-offs. J Intern Med 275:317-330. https://doi.org/10.1111/joim.12148

26. Reitan RM (1955) The relation of the trail making test to organic brain damage. J Consult Psychol 19:393-394

27. Resnick SM, Pham DL, Kraut MA, Zonderman AB, Davatzikos C (2003) Longitudinal magnetic resonance imaging studies of older adults: a shrinking brain. J Neurosci 23:3295-3301

28. Serrano-Pozo A, Qian J, Monsell SE, Blacker D, Gomez-Isla T, Betensky RA, Growdon JH, Johnson KA, Frosch MP, Sperling RA et al (2014) Mild to moderate Alzheimer dementia with insufficient neuropathological changes. Ann Neurol 75:597-601. https://doi.org/10.1002/ana.24125

29. Serrano-Pozo A, Qian J, Monsell SE, Frosch MP, Betensky RA, Hyman BT (2013) Examination of the clinicopathologic continuum of Alzheimer disease in the autopsy cohort of the National Alzheimer Coordinating Center. J Neuropathol Exp Neurol 72:1182-1192. https://doi.org/10.1097/ NEN.0000000000000016

30. Teylan M, Besser LM, Crary JF, Mock C, Gauthreaux K, Thomas NM, Chen YC, Kukull WA (2019) Clinical diagnoses among individuals with primary agerelated tauopathy versus Alzheimer's neuropathology. Lab Investig. https://doi.org/10.1038/s41374-019-0186-0

31. Wechsler D. (1939) The measurement of adult intelligence. Baltimore: The Williams \& Wilkins Company.

\section{Publisher's Note}

Springer Nature remains neutral with regard to jurisdictional claims in published maps and institutional affiliations.

Ready to submit your research? Choose BMC and benefit from:

- fast, convenient online submission

- thorough peer review by experienced researchers in your field

- rapid publication on acceptance

- support for research data, including large and complex data types

- gold Open Access which fosters wider collaboration and increased citations

- maximum visibility for your research: over $100 \mathrm{M}$ website views per year

At BMC, research is always in progress.

Learn more biomedcentral.com/submissions 